\title{
Transition Dipole Moments of One-Dimensional Excitons in Soluble Phthalocyanine Thin Films
}

\author{
Libin Liang ${ }^{\dagger}$, KimNgan Burrill ${ }^{\dagger, \|}$, and Madalina I. Furis*,†, \\ ${ }^{\dagger}$ Materials Science Program and Department of Physics, University of Vermont, 82 University Place, \\ Burlington, Vermont 05405, United States \\ "Intel Corporation, Rio Rancho, NM 87124, United States

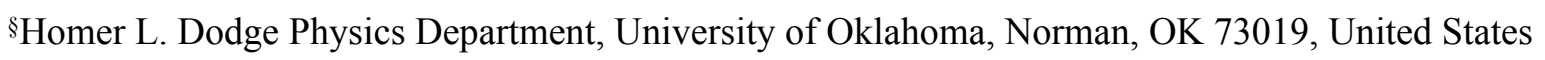 \\ *Email: madalina.furis@ou.edu. Tel.: (405) 9342221
}

\section{1. $\mathrm{H}_{2}-\mathrm{OBPc}$ thin film preparation with solution-based pen writing (PW) technique}

The schematic of the pen writing technique is shown in

Figure S1. Solution of $0.5 \%$ by weight $(0.5 \mathrm{mg} / 100 \mu \mathrm{L})$ was prepared by dissolving $\mathrm{H}_{2}-\mathrm{OBPc}$ in toluene. Solution was then loaded into a hollow capillary tube $\left(0.6 \times 6 \mathrm{~mm}^{2}\right)$ and held inside by capillary forces. Capillary tube was mounted vertically on a fixed stage. Substrate was placed on a computer-controlled onedimensional translation stage (Zaber Technologies). The capillary tube was then lowered down to approach the substrate until the solution meniscus came in contact with the c-plane round-shaped single-crystal sapphire substrate. The translation stage was then moved with constant speed (writing speed) controlled by computer. A thin layer of OBPc was deposited on the substrate as the solution evaporated. Under optimum conditions, ordered thin film with macroscopic grain size and high crystallinity could be obtained as shown in paper (Figure 1). More information about the various thin film growth regimes is discussed in reference $\mathrm{x}$ of the main paper.

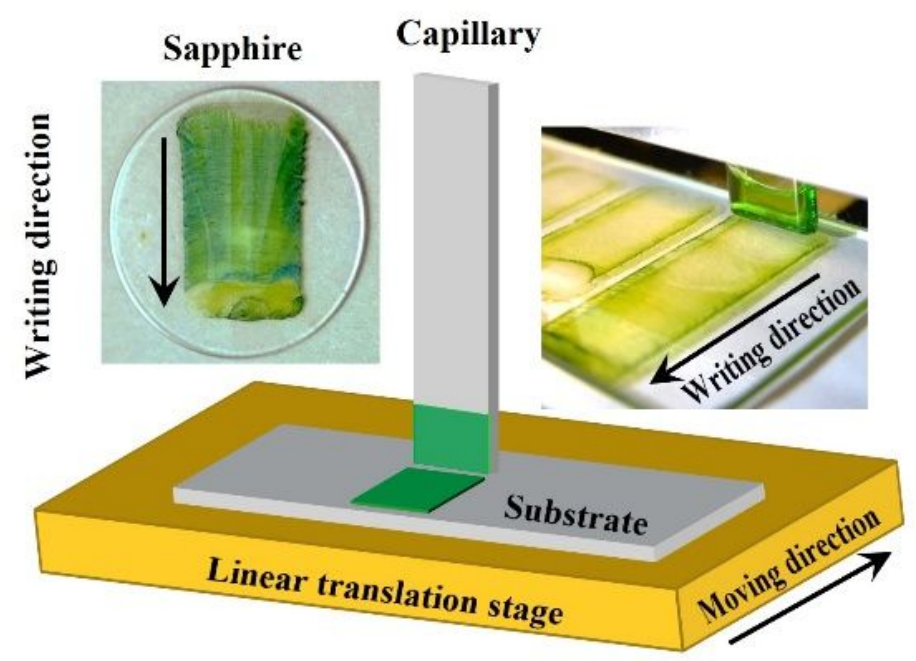

Figure S1. Schematic of pen writing setup 


\section{Incident light $\vec{K}$-vector dependent absorption experiment}

Figure S2 depicts the absorption experiment setup built on an optical table with free-space optics. For the normal incidence, light propagation direction is parallel with the normal direction to the thin film surface. A quasi-monochromatic tunable beam was prepared using a tungsten lamp and coupled to a monochromator from Spectral Products. Incident light was vertically polarized. Its polarization was modulated between orthogonal directions at $100 \mathrm{kHz}$ using a photoelastic modulator (PEM, Hinds Instruments) and then rotated I the $\mathrm{x}-\mathrm{y}$ (substrate plane) using by a variable angle $\alpha$ using a half waveplate (HWP). Incident light was focused on the sample with the aid of a long distance in-situ telescope that monitored the spot location within a crystalline grain. Transmitted signal was then collected by a Si detector using lock-in technique to improve signal-to-noise ratio. That is, for average absorption measurement, the incident light is modulated to be in on-off mode by a mechanic chopper with frequency of $270 \mathrm{~Hz}$. For Linear dichroism (LD) measurements, the polarization of the incident light is modulated to alternative between orthogonal directions by the PEM with frequency of $100 \mathrm{kHz}$. Therefore, the transmitted raw signal $T_{i}$ contains low frequency signal VDC $(270 \mathrm{~Hz})$ and high frequency signal VAC $(100 \mathrm{kHz})$. The LD was then calculated from these values using the method reported in a previous study ${ }^{1}$.

Other incident light $\vec{K}$-vector orientations shown in figure 1a of the main paper were achieved with the following approach. The $\vec{K}\left(\mathrm{x}-\mathrm{z}-30^{\circ}\right)$ incident light orientation was first obtained by performing the rotation operation $\mathrm{R}_{\mathrm{y}}\left(+30^{\circ}\right)$ to the sample holder (Figure $\mathrm{S} 2 \mathrm{~d}$ ). That is, rotating the sample holder by $30^{\circ}$ with respect to y axis. The $\vec{K}\left(\mathrm{x}-\mathrm{z}-30^{\circ}\right), \vec{K}\left(\mathrm{x}-\mathrm{z}+30^{\circ}\right)$ and $\vec{K}\left(\mathrm{y}-\mathrm{z}+30^{\circ}\right)$ incident light orientations (Figure S2a, c and b, respectively) were then obtained by rotating the round-shape sapphire substrate count-clockwise by $90^{\circ}$, $180^{\circ}$ and $270^{\circ}$, which are equivalent to performing the rotation operation $R_{x}\left(-30^{\circ}\right), R_{y}\left(-30^{\circ}\right)$ and $R_{x}\left(+30^{\circ}\right)$ to the sample holder with respect to the $\vec{K}$ (normal) orientation, respectively. 


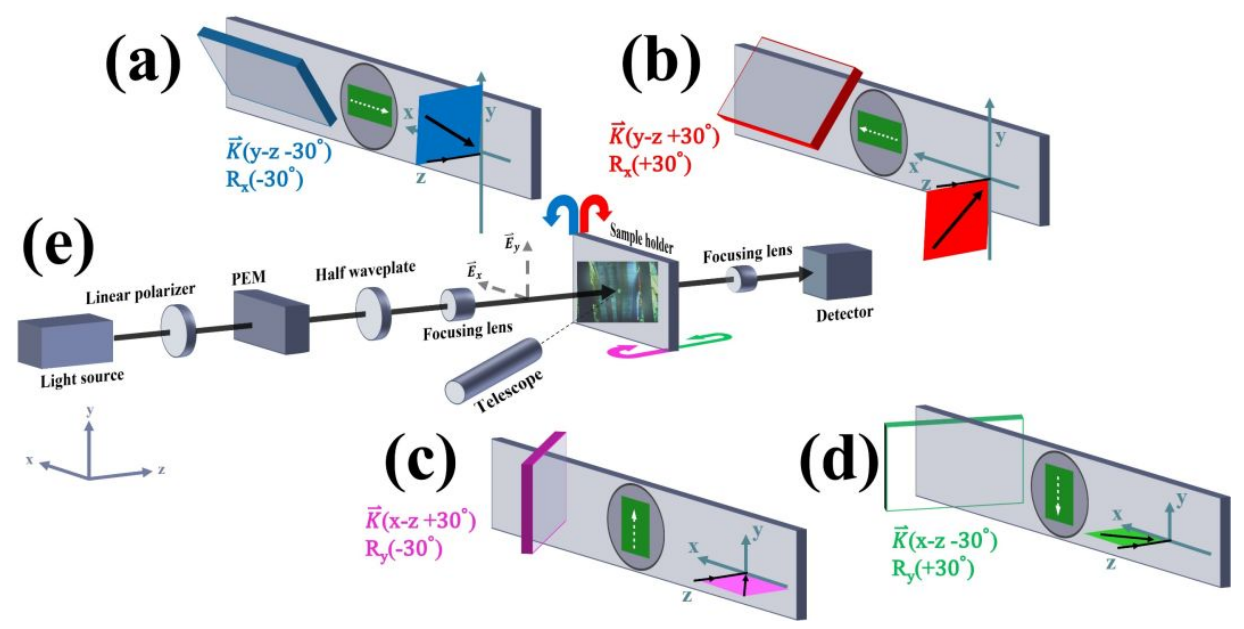

Figure S2. Schematic of the incident light $\overrightarrow{\boldsymbol{K}}$-vector-dependent absorption experiment setup. (e) The absorption setup with normal incident light orientation. (a)-(d) The other incident light orientations. On the left-hand side of each geometry image are the equivalent rotations of the sample holder with respective to the $\overrightarrow{\boldsymbol{K}}$ (normal), the center depicts the actual rotation of the round-shape sapphire on sample holder, while the right-hand side shows the illustrations of the incident light orientation with respect to the sample, color coded similarly to Figure 1 in the main text. 


\section{Treatment of raw transmitted signal in absorption measurements.}

The absorbance is computed in the conventional way, i.e., $A_{i}=-\ln \left(T_{i} / T_{0}\right)$, where $\mathrm{A}_{\mathrm{i}}$ is the absorbance at wavelength of $\mathrm{i}-\mathrm{nm}, \mathrm{T}_{\mathrm{i}}$ is the transmitted electronic signal after sample at wavelength of $\mathrm{i}-\mathrm{nm}$, and $\mathrm{T}_{\mathrm{o}}$ is the total electronic signal before sample. The OBPc PW thin films contains macroscopic grains that consist of parallel stripes with gaps in between (see SEM image). Such structure would create an interference pattern similar to a diffraction grating. Consequently, interference patterns are observed in VAC and VDC transmitted signal (Figure S3a and b) with linearly polarized incident light. In contrast, no interference pattern is observed in PW thin film with unpolarized incident light, or in SC thin film with polarized incident light. This interference effect would distort absorption spectra with alternative constructive and destructive patterns, especially for the intense absorption peaks around $850 \mathrm{~nm}$ (typical spectrum was shown in Figure $\mathrm{S} 3 \mathrm{c})$. To eliminate this artifact effect in absorption and LD measurements, $T_{i}$ is collected in the following way. For the transmitted signal polarized at an angle $\alpha$ with respect to the $\mathbf{x}$ - axis, the signal $\mathrm{T}\left(\alpha \pm 30^{\circ}\right)$ and $\mathrm{T}\left(\alpha \pm 60^{\circ}\right)$ are also detected. Then the average signal T(average) is computed as

$$
T(\text { average })=\frac{T\left(\alpha+30^{\circ}\right)+T\left(\alpha-30^{\circ}\right) \pm T\left(\alpha+60^{\circ}\right) \pm T\left(\alpha-60^{\circ}\right)}{4 \times \cos \left(30^{\circ}\right)}
$$

where the plus sign is for VDC transmitted signal in absorbance calculation, since the VDC signal are all positive values; the minus sign is for VAC transmitted signal in LD calculation, since the VAC signal would switch sign every $90^{\circ}$; the division of $\cos \left(30^{\circ}\right)$ is for value correction since the measurements are conducted $30^{\circ}$ off the direction $\alpha$. In this approach, smooth absorption curves could be restored with accuracy (for example the average curves in Figure S3).

(a)

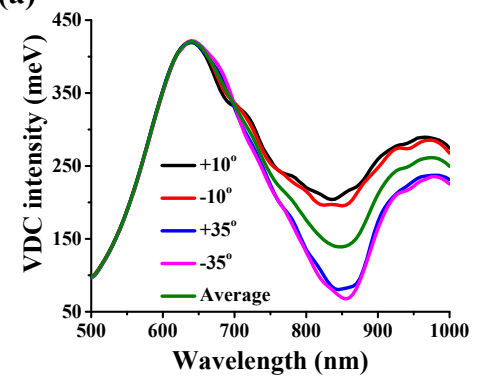

(b)

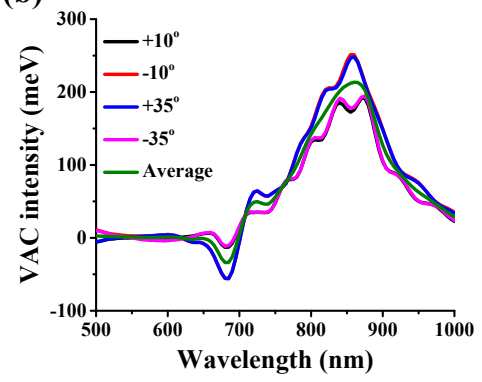

(c)

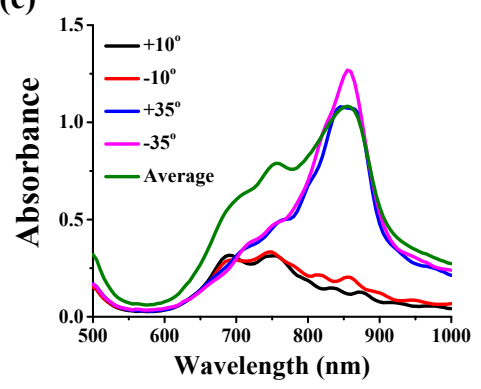

Figure S3. Typical spectra of transmitted signal with interference and spectra after averaging. (a) VAC signal in LD measurements, (b) VDC signal in absorption measurements and (c) Angular absorption spectra. 


\section{Incident $\vec{K}$-vector orientation-dependent absorption polar plots from experiment and simulation.}

Incident light $\vec{K}$-vector orientation-dependent absorption polar plots from experiment were generated with the following procedure: (A) Absorption and LD spectra were first measured with HWP rotated by $\alpha / 2$ (i.e., incident light polarization is rotated by $\alpha$ in $\mathrm{x}-\mathrm{y}$ plane). (B) Peak fit deconvolution was conducted upon the spectra to identify the transition wavelength and extract the peak intensity values. Angular absorbance was then calculated as discussed in previous section. (C) Step (A) and (B) were repeated with $\alpha$ ranging from $0^{\circ}$ to $90^{\circ}$ in step of $10^{\circ}$. (D) Step (A)-(C) were repeated with a different incident $\vec{K}$-vector orientation.

Incident light $\vec{K}$-vector dependent absorption polar plots from simulation were generated for comparison. The Cartesian coordinates in laboratory $(\mathrm{x}, \mathrm{y}, \mathrm{z})$ and for each transition dipole $\left(x^{\prime}, y^{\prime}\right.$ and $\left.z^{\prime}\right)$ are shown in Figure S4a. At $\vec{K}$ (normal) orientation, following the procedure presented by Flora et al. ${ }^{2}$, the dipole components $(\vec{\mu})$ in lab axis could be expressed as

$$
\vec{\mu}(\text { normal })=\left[\mu_{x}, \mu_{y}, \mu_{z}\right]^{T}=R_{z}(\varphi) \cdot R_{x}(\theta) \cdot \overrightarrow{\mu^{\prime}}
$$

The general form of a linearly polarized transition dipole is represented by its three components $\mu_{x^{\prime}}, \mu_{y^{\prime}}$ and $\mu_{z^{\prime}}$ along the coordinate axises given by $\vec{\mu}=\left[\mu_{x^{\prime}}, \mu_{y^{\prime}}, \mu_{z^{\prime}}\right]^{T}$. An elliptically polarized transition dipole is given as $\vec{\mu}^{\prime}=\left[\mu_{x^{\prime}}, \cdot \cdot \mu_{y^{\prime}}, 0\right]^{T}$. Transition dipoles observed at other $\vec{K}$-vectors, $\vec{\mu}\left(\mathrm{y}-\mathrm{z} \pm 30^{\circ}\right)$ and $\vec{\mu}\left(\mathrm{x}-\mathrm{z} \pm 30^{\circ}\right)$ could be explored by rotating sample holder. This suggests another rotation operation to the dipole with respect to $\mathrm{x}$ or y axes (shown in Figure S2). Therefore, the dipole components in lab axis could be expressed as

$$
\vec{\mu}\left(y-z \pm 30^{\circ}\right)=R_{x}\left( \pm 30^{\circ}\right) \cdot \vec{\mu}(\text { normal }), \vec{\mu}\left(x-z \pm 30^{\circ}\right)=R_{y}\left(\mp 30^{\circ}\right) \cdot \vec{\mu}(\text { normal })
$$

To obtain the polarization-dependent absorbance, a horizontally polarized electric field $\vec{E}=[1,0,0]$ was applied to each dipole, and the absorbance value per unit volume computed as $A=|\vec{E} \cdot \vec{\mu}|^{2}$. This would give the angular absorbance value along horizontal direction. Notice that under the tightest-focus condition of the incident light, for incident $\vec{K}$ (others) orientation ( $\vec{K}$-vector orientations except $\vec{K}$ (normal)), the total volume that the incident go through the thin film sample is increased by $1 / \cos 30^{\circ}$ as shown in Figure S4a. Finally, the angular absorbance is expressed as

$$
A(\alpha)=\left\{\begin{array}{l}
\left|R_{z}(\alpha) \cdot \vec{E} \cdot \vec{\mu}(\vec{K})\right|^{2}, \vec{K}=\vec{K}(\text { normal }) \\
\left|R_{z}(\alpha) \cdot \vec{E} \cdot \vec{\mu}(\vec{K})\right|^{2} / \cos 30^{\circ}, \vec{K}(\text { others })
\end{array}, \text { with } \alpha \text { from } 0^{\circ} \text { to } 360^{\circ}\right. \text { (4) }
$$


Therefore, absorbance simulation plot could be generated with the above equation, by calculating $A(\alpha)$ and plotting $A(\alpha)$ versus $\alpha$, for any orientations of electric field vectors or transition dipoles. The excitonic transition dipole orientation could be estimated by comparing the incident light $\vec{K}$-vector orientationdependent absorption polar plots from experiment and those from simulation.
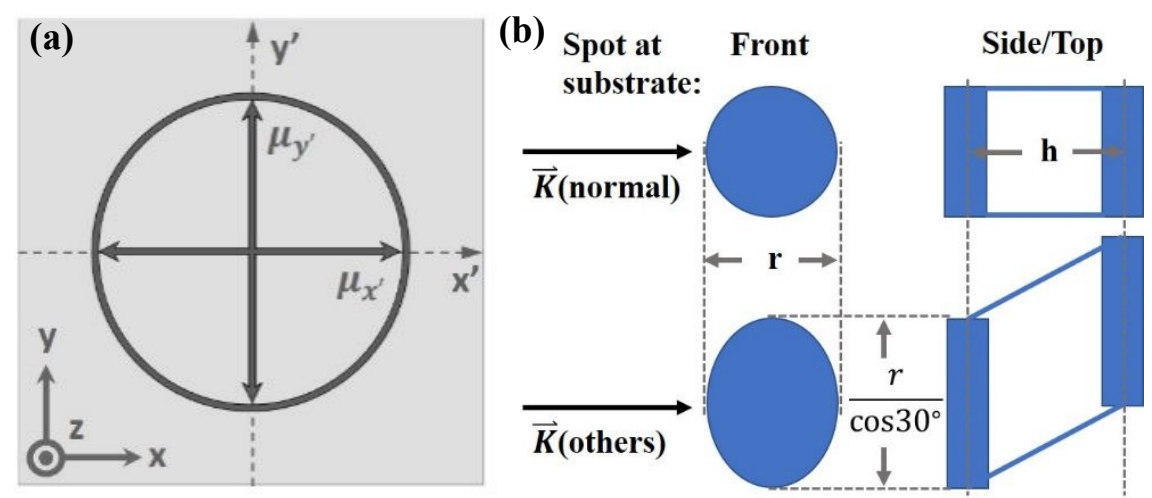

Figure S4. Transition dipole orientation on substrate. (a) Coordinate system: $(\mathrm{x}, \mathrm{y}, \mathrm{z})$ for lab axis and $\left(x^{\prime}, y^{\prime}\right.$ and $\left.z^{\prime}\right)$ for transition dipole axis, represented by the dipole component $\mu_{x^{\prime}}$ and $\mu_{y^{\prime}}, \mu_{z^{\prime}}$ is not shown for clarity. (b) Shape of of the tightest-focus incident light beam on substrate at $\vec{K}$ (normal) and $\vec{K}$ (others), where $\mathrm{r}$ is radius of the focused beam spot and $\mathrm{h}$ is the thickness of the thin film. 


\section{Peak fit analysis in absorption and Magnetic Circular Dichroism (MCD) spectra}

Figure S5 shows the typical peak fit results. $685 \mathrm{~nm}$ and $755 \mathrm{~nm}$ peak are assigned as the HOMO-n to LUMO transitions of the OBPc PW thin film. Consequently, for $685 \mathrm{~nm}$ peak, MCD spectrum (Figure S5a) shows the negative contributions (M1 and M2). Both absorption (Figure S5b) and LD spectra (Figure S5c) show obvious peaks around $685 \mathrm{~nm}$ (A1 and L1). For $755 \mathrm{~nm}$ peak, MCD spectrum shows the Faraday A term feature with a negative peak (M3) and a positive peak (M4), centering at $755 \mathrm{~nm}$. Absorption spectrum shows a strong absorption peak (A3) while LD spectrum shows a small peak (L3). Additional peaks with low intensity at around $700 \mathrm{~nm}$ peak were employed to in absorption and LD spectra (A2 and L2, respectively) to account for the broadening of the spectrum. Similar peak fit results could be found in earlier studies $^{3-5}$. As discussed above, transition states centered from $800 \mathrm{~nm}$ to $850 \mathrm{~nm}$ are the result of long-range intermolecular interaction and originate from non-degenerate transition dipoles from molecule. Therefore, we fit the $855 \mathrm{~nm}$ broad peaks in MCD, absorbance and LD spectra with two peaks centered at around 835 $\mathrm{nm}$ (M6, A5 and L5) and $865 \mathrm{~nm}$ (M7, A6 and L6). $800 \mathrm{~nm}$ peaks are also employed to account for the shoulder of the $855 \mathrm{~nm}$ broad peaks in absorbance and LD (A4 and L4), and the intense peak in MCD (M5). MCD spectrum shows no obvious peak or shoulder for wavelengths longer than $865 \mathrm{~nm}$ (M7), but $920 \mathrm{~nm}$ peaks are still introduced to fit the tail of the $855 \mathrm{~nm}$ broad peak in absorbance and LD spectra (A7 and L7). Therefore, $800,835,865$ and $920 \mathrm{~nm}$ are assigned as the excitonic transition states predicted by factor group splitting arising from intermolecular interaction. Peak fit deconvolution is performed to all spectra at different $\vec{K}$-vectors and peak energies s are kept constant between spectra.

(a)

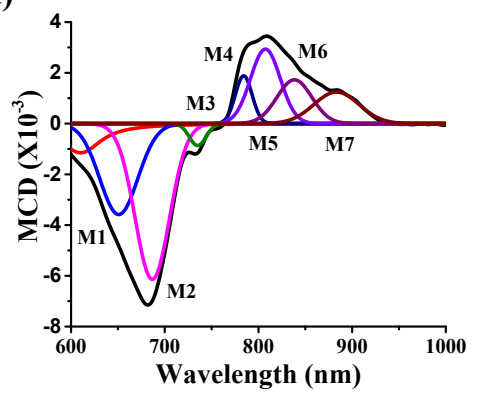

(b)

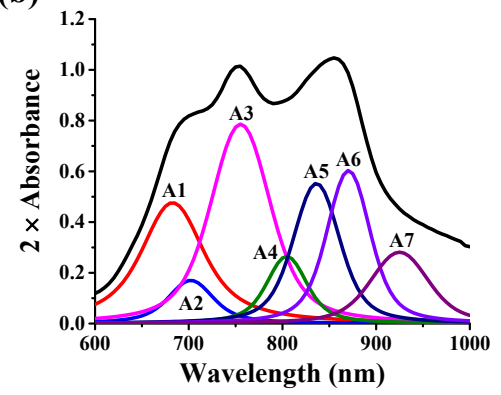

(c)

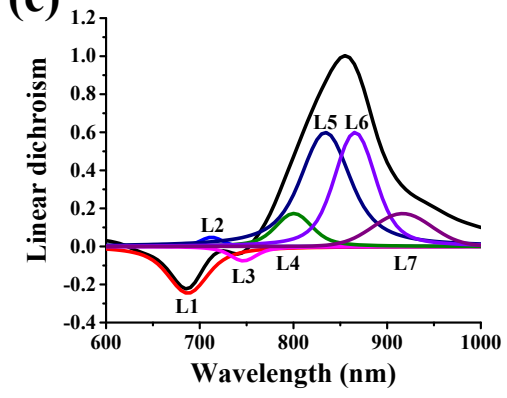

Figure S5. Typical peak fit results for spectra from $\mathrm{H}_{2}-\mathrm{OBPc} \mathrm{PW}$ thin films. (a) MCD spectrum (4 K). (b) absorption spectrum. (c) LD spectrum. 


\section{Incident light $\vec{K}$-vector orientation-dependent absorption polar plots of $835 \mathrm{~nm}$ dipole at room}

temperature.

Figure S6 shows the incident light $\vec{K}$-vector orientation-dependent absorption polar plots of $835 \mathrm{~nm}$ dipole at room temperature. It has similar feature as those of $865 \mathrm{~nm}$ dipole. Figure $\mathrm{S} 6$ and Figure 4 in the main text form the complete set of incident light $\vec{K}$-vector orientation-dependent absorption polar plots of transition dipoles in $\mathrm{H}_{2}-\mathrm{OBPc} \mathrm{PW}$ thin films at room temperature.
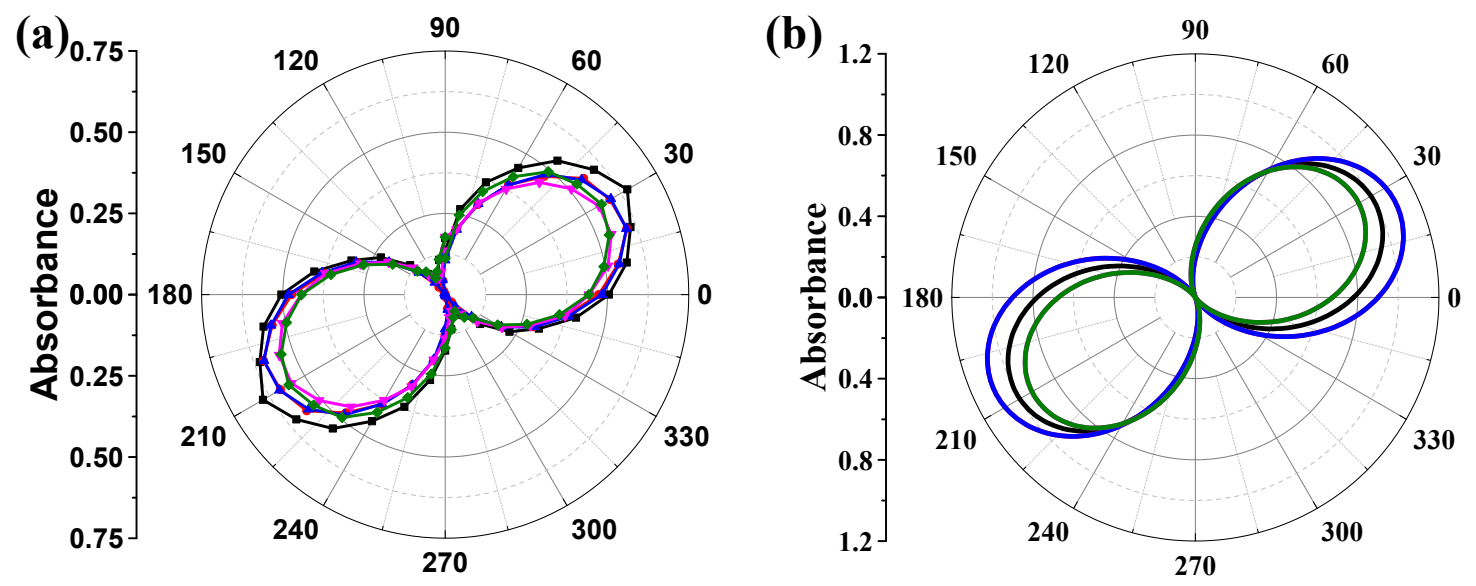

Figure S6. Angular absorbance plots of $835 \mathrm{~nm}$ dipole at room temperature. (a) from experiment and (b) from simulation. Black curves are for $\vec{K}$ (normal), red for $\vec{K}\left(\mathrm{y}-\mathrm{z}+30^{\circ}\right)$, blue for $\vec{K}\left(\mathrm{y}-\mathrm{z}-30^{\circ}\right)$, pink for $\vec{K}\left(\mathrm{x}-\mathrm{z}+30^{\circ}\right)$ and green for $\vec{K}\left(\mathrm{x}-\mathrm{z}-30^{\circ}\right)$. 


\section{Incident light $\vec{K}$-vector orientation-dependent absorption polar plots at $4 \mathrm{~K}$.}

Figure S7 shows the complete set of incident light $\vec{K}$-vector orientation-dependent absorption polar plots of transition dipoles in $\mathrm{H}_{2}-\mathrm{OBPc} \mathrm{PW}$ thin films at $\mathrm{T}=4 \mathrm{~K}$. The polar plots exhibit similar feature with those obtained at room temperature for each excitonic transition dipole (Figure S6 and Figure 4 in paper). The additional excitonic transition dipole $900 \mathrm{~nm}$ at $4 \mathrm{~K}$ has similar pattern with $835 \mathrm{~nm}$ and $865 \mathrm{~nm}$ dipoles, which implies it has similar orientation with the $835 \mathrm{~nm}$ and $865 \mathrm{~nm}$ dipoles.
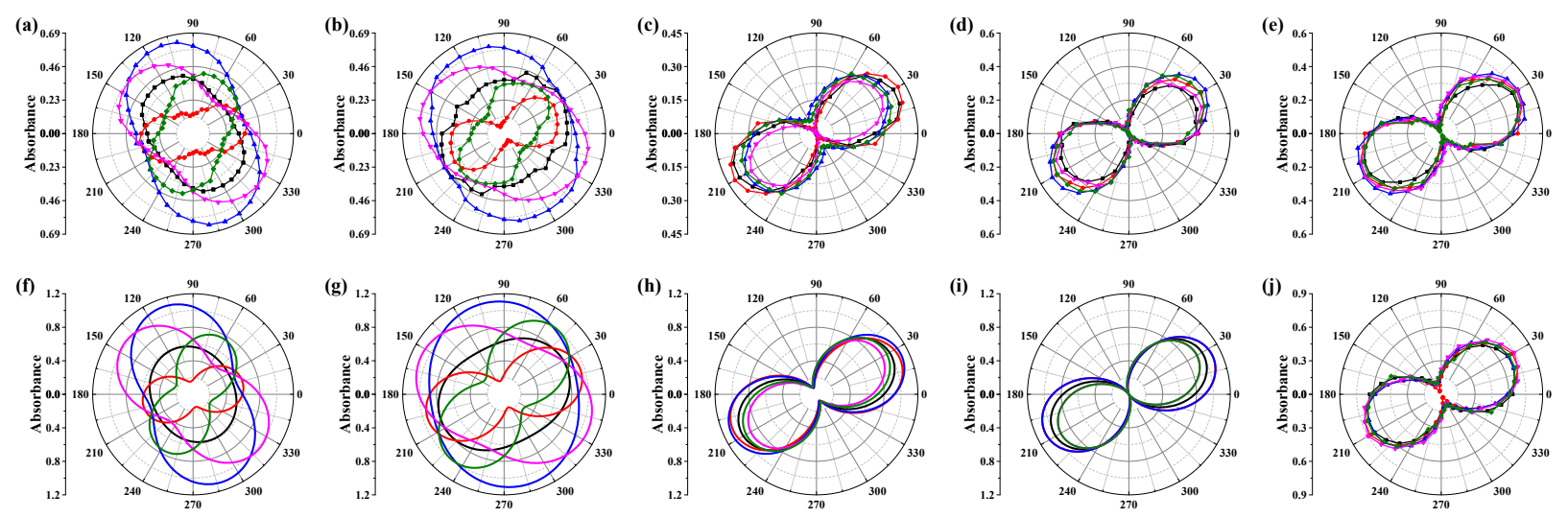

Figure S7. Angular absorbance plots at 4 K. (a), (b), (c), (d), (e), (j) are $685 \mathrm{~nm}, 755,800,835,865$ and $900 \mathrm{~nm}$ from experiment, respectively. (f), (g), (h) are 685, 755 and $800 \mathrm{~nm}$ from simulation, respectively. (i) is 835,865 and $900 \mathrm{~nm}$ from simulation. Black curves are for $\vec{K}$ (normal), red for $\vec{K}\left(\mathrm{y}-\mathrm{z}+30^{\circ}\right)$, blue for $\vec{K}$ (y-z $\left.-30^{\circ}\right)$, pink for $\vec{K}\left(\mathrm{x}-\mathrm{z}+30^{\circ}\right)$ and green for $\vec{K}\left(\mathrm{x}-\mathrm{z}-30^{\circ}\right)$. 


\section{Raman measurements on $\mathrm{H}_{2}$-OBPc thin film at room temperature}

$\mathrm{H}_{2}$-OBPc PW thin film was deposit on a glass slide substrate with writing speed of $17 \mu \mathrm{m} / \mathrm{s}$. Raman measurements were conducted with HORIBA LabRam HR Evolution confocal Raman microscope using an incident laser of wavelength $532 \mathrm{~nm}$. An ultra-low frequency filter was employed to allow Raman measurement below $100 \mathrm{~cm}^{-1}$ (in present work, lowest Raman frequency accessible was $50 \mathrm{~cm}^{-1}$ ). The low frequency lattice vibration (phonon) mode $80.6,165.0$ and $268.6 \mathrm{~cm}^{-1}$ that are believed to be responsible for the intermolecular coupling and the formation of the delocalized polarons are clearly present. The molecular vibration mode at $1603.5 \mathrm{~cm}^{-1}$ is also detected, which can be described as the collective stretching mode of $\pi$-conjugated backbone and is responsible for localizing the excitons.

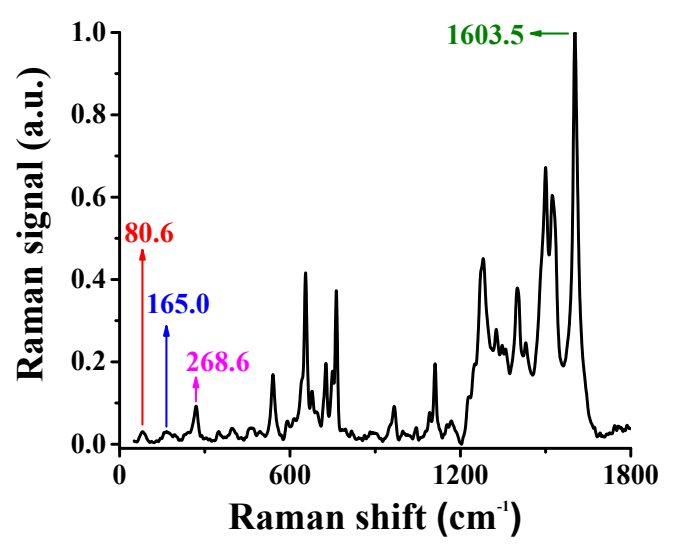

Figure S8. Raman spectra of $\mathrm{H}_{2}-\mathrm{OBPc} \mathrm{PW}$ thin film at room temperature 


\section{Reference}

1. Drake, A. F., Polarisation Modulation-the Measurement of Linear and Circular Dichroism. Journal of Physics E: Scientific Instruments 1986, 19, 170.

2. Flora, W. H.; Mendes, S. B.; Doherty, W. J.; Saavedra, S. S.; Armstrong, N. R., Determination of Molecular Anisotropy in Thin Films of Discotic Assemblies Using Attenuated Total Reflectance UvVisible Spectroscopy. Langmuir 2005, 21, 360-368.

3. Rawat, N. Exchange Mechanisms in Macroscopic Ordered Organic Magnetic Semiconductors. University of Vermont, 2015.

4. Hollebone, B. R.; Stillman, M. J., Assignment of Absorption and Magnetic Circular Dichroism Spectra of Solid, A Phase Metallophthalocyanines. Journal of the Chemical Society, Faraday Transactions 2: Molecular and Chemical Physics 1978, 74, 2107-2127.

5. Kobayashi, N.; Ogata, H.; Nonaka, N.; Luk'yanets, E. A., Effect of Peripheral Substitution on the Electronic Absorption and Fluorescence Spectra of Metal-Free and Zinc Phthalocyanines. Chemistry-A European Journal 2003, 9, 5123-5134. 